\title{
Design of Laser Ranging System Based on Stm32
}

\author{
Yu-jie Chen ${ }^{1, a}$, Pei-xue Liu ${ }^{1, b}$, Hui-wei Chen ${ }^{1, c}$, Shu-mei Liu ${ }^{1, d}$ \\ Qingdao Huanghai College, Qingdao 266427, China \\ achenyujie_cc@163.com, b 93987898 @qq.com \\ c $83430761 @ q q . c o m,{ }^{d} 1309889426 @ q q . c o m$
}

Keywords: Laser ranging, Single-chip, Voice broadcast, Liquid crystal display

\begin{abstract}
Laser ranging is a widely used laser technology. It has many advantages, such as convenient operation, high precision, and the ability to use it anytime and anywhere. In this paper, a portable laser ranger based on STM32 is designed, which can perform high-precision ranging function under complicated working conditions. The system is composed of 4 modules, including STM32 module, laser ranging module, WT588D voice module and TFT LCD module. The system also measures the time of laser flight (and the phase difference of the echo), and the measurement results can be accurate to thousands of points, thus ensuring the accuracy and reliability of the ranging.
\end{abstract}

\section{Introduction}

With the development of society, distance measurement plays an important role in aviation, military, construction and other fields. Laser has the characteristics of good monochromaticity, good coherence, strong brightness and directional, which makes the laser range finder come into our sight and developed rapidly. It gets widely used because laser rangefinder has characteristics of small size, light weight and easy to carry. So the study of laser ranging technology is very meaningful.

\section{Overall System Design}

The main purpose of this paper is to design a portable laser range finder based on STM32 single chip microcomputer. The main working process is to measure the distance between person and obstacle by laser distance measurement module and send

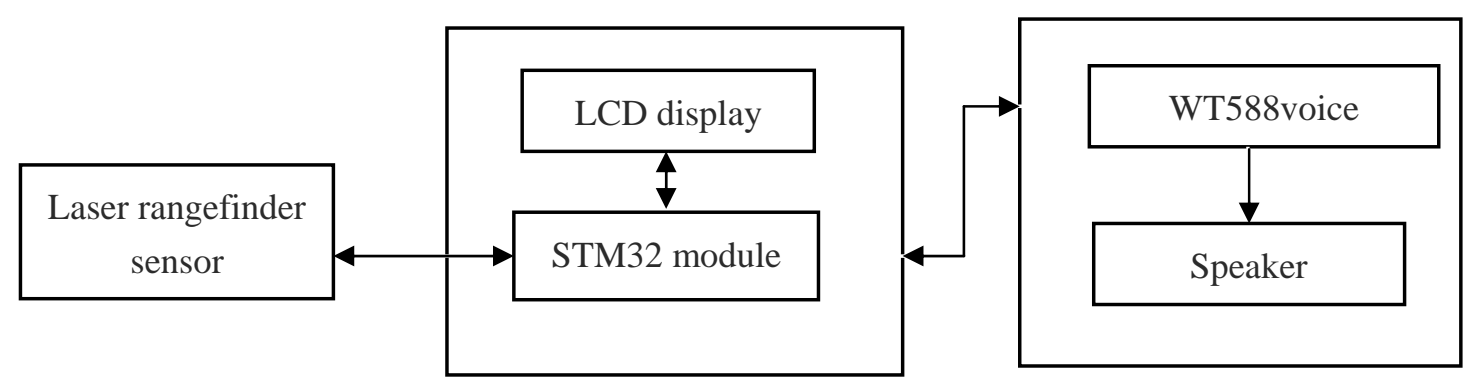

Figure 1 block diagram of the system structure

The measured distance to the controller. The controller controls The LCD module and the voice broadcast module make it possible to display and broadcast the measured distance.The 
system is mainly composed of laser ranging sensor, TFT liquid crystal display module, STM32 single chip microcomputer system, WT588 voice broadcast module, the system structure block diagram shown in Figure 1.

\section{Hardware Design}

Display circuit module

The design of the display circuit, select the TFT LCD screen, TFT LCD screen is a thin film transistor, is now the PC, TV and other major display devices. In the display screen integrates a large number of transistors, used to drive the LCD screen on each pixel, so TFT LCD screen display color and brightness are very good. In this design, in order to save IO resources, but also taking into account the amount of data and TFT communication is not much, so consider the use of serial communication TFT screen way.

The design is mainly using the TFT display to display the measured distance. The design problem in hardware is focused on doing the TFT display interface. Where we choose the serial communication, but also to the TFT display backlight for 3.3V power.

Voice broadcast module

The voice design module is used WT588D-U voice module, WT588D voice module is a powerful repeatable erase programming voice module. Supporting WT588D VoiceChip PC operating software can be free to replace the WT588D voice module of any one control mode, the information can be downloaded to the SPI-Flash. Software operation is simple and easy to understand, the integration of the voice combination technology, greatly reducing the voice editing time, fully support online download, even if the WT588D power case, the same can be downloaded to the associated SPI-Flash download information, to WT588D Voice module circuit reset, you can update to just download the control mode.

Install the WT588D-U voice module using the Voice Chip software, the need to create a new project, and then have prepared the voice loaded into the software, then drag the voice inside the address, and then set the project in the project set up a serial port control mode, BUSY output status is low, the last point of the compiler to compile the entire project. Use the USB cable to connect the host computer to the voice module and download the engineering information to the WT588D-U module. WT588D-U voice module application block diagram shown in Figure 2.

Laser ranging module

Laser ranging module a general block diagram of the pulse laser ranging system, the system consists of five parts: laser transmitter unit, a receiving channel, signal acquisition module, signal modulation module and data control processing module. The laser emitting unit transmits the laser pulse at time t, passes through the diffuse reflection of the target, returns to the receiving module of the laser range finder, receives the return pulse from the receiving channel through the receiving optical system, passes through the filtering and amplifies the signal to the signal acquisition module , The signal acquisition module, the signal modulation module sampling the high-frequency signal processing, the measured signal after the time domain to expand and compare processing, and finally output to the data control processing unit, get the distance $\mathrm{S}$ 


\section{Software Design}

System flow chart

When the laser range finder is connected to the power after the first initialization, initialization successful broadcast welcome to use the laser range finder, and display on the display reading, and then display the measured distance, when pressed, the laser range finder began to move, Laser emitting diodes to emit laser, to reach the object when the laser reflected back, reflected back through the data processing laser, the exact distance. At the same time, the display and voice broadcast module also began to action, the LCD display distance, voice broadcast module broadcast shows the distance.

Flow chart of laser ranging module

When the laser range finder just connected to the power, the laser range finder initialization, began to emit the laser, the laser encountered obstacles after the reflection back, laser range finder laser receiving module to receive reflected back to the laser, through the mixing sampling technology The distance between the position of the laser rangefinder and the position of the obstacle. When the laser range finder has been connected to the power again after measuring the distance need to press the key0, laser range finder re-initialization, repeat the process. The laser ranging module is shown in Figure 2.

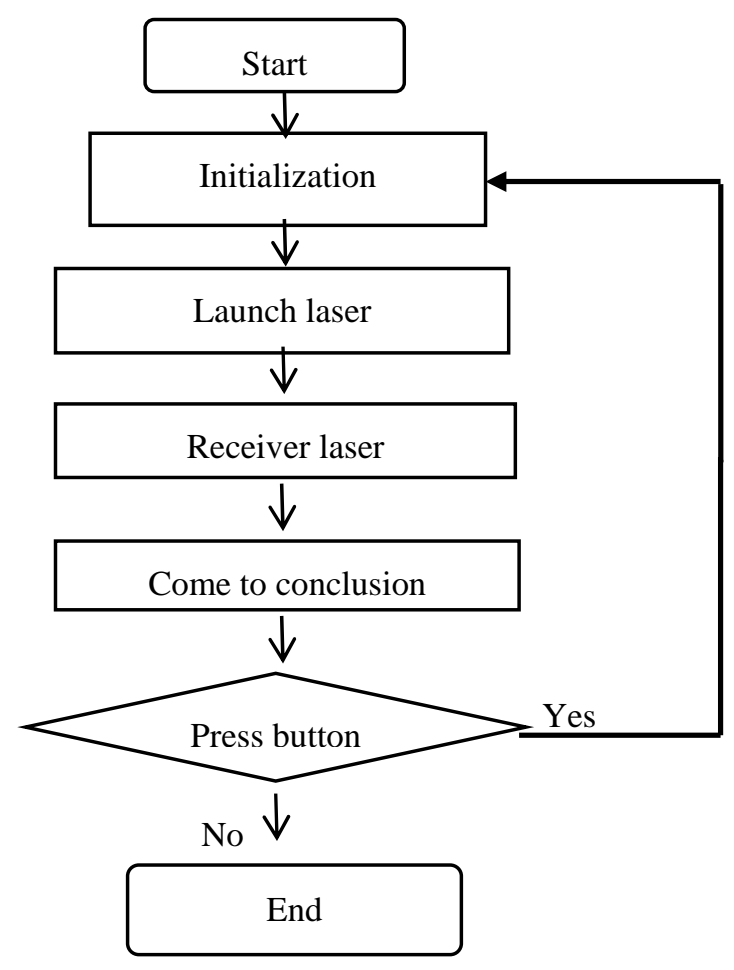

Figure 2 Flow chart of the laser ranging module

\section{Voice broadcast module}

Voice broadcast module is used WT588U chip, the chip has a large capacity ROM can store sound files. The voice module is written in the corresponding position by writing an address operation in advance. In order to facilitate the programming, the design broadcast broadcast "1" voice corresponding to the address, "2" voice broadcast 2 address, and so on, 
the use of serial communication protocol WT588U module address write operation, after receiving the write signal, WT588U The chip will automatically identify the address, remove the address of the audio signal, according to the tone of the audio release of different frequencies of the PWM waveform driver speakers, playing the voice.

When the voice broadcast first to determine the location of the highest position, because the design of the range of about 30 meters range, so only need to determine the highest bit is ten or a bit, when the judge is the highest bit is ten, broadcast need to The number followed by "ten", and then continue to broadcast a single digit, if the highest digit is a bit, then only broadcast the number, broadcast the number after the broadcast "point", and then broadcast the decimal point followed by the number, the design The distance from the accurate to the decimal point behind the three, so broadcast after the decimal point behind the three need to continue to broadcast "rice" to complete the broadcast.

\section{System Test}

To debug the system, you should first test the feasibility of the minimum system, the voice module and the laser distance measurement module, and make sure that each module is accurate and assembled, and the hardware module of the laser range finder is assembled. Second, the laser range finder program debugging, to determine the program without grammatical errors, the program will be downloaded through the emulator to the laser range finder control chip, the laser range finder debugging.

The distance between the person's hand and the ground is measured by a laser range finder. The measurement result is 1.252 meters. The requirements of the three requirements in the task book are accurate to the decimal point. The laser range finder broadcasts this distance and broadcasts it on the LCD screen The display is shown in Figure 3.

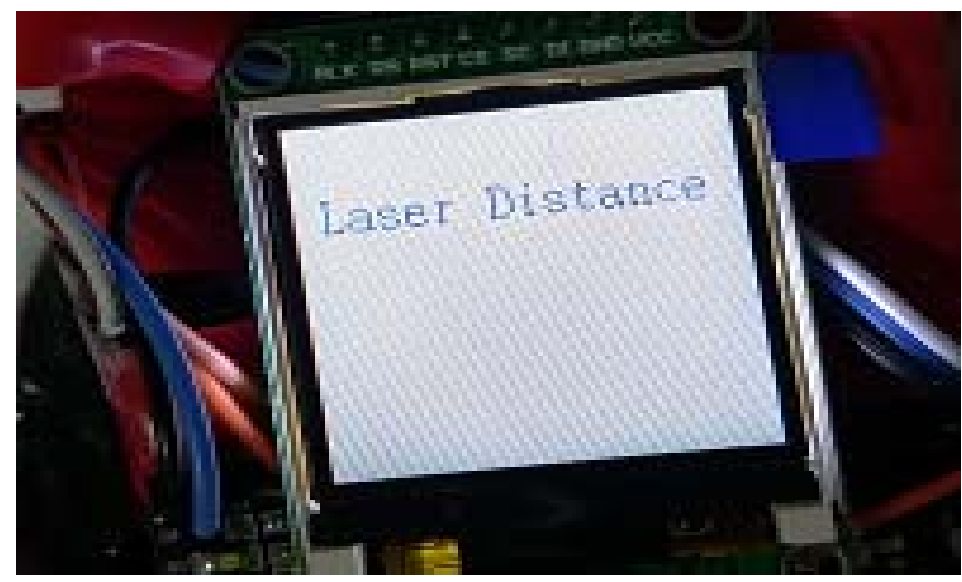

Figure 3 Distance display

\section{Conclusion}

This paper designs a portable laser range finder based on STM32 microcontroller microcontrollers. The design uses STM32F103C microcontroller as the main control chip control laser ranging module and voice broadcast module and liquid crystal display module. The STM32F103C microcontroller controls the laser rangefinder to emit lasers when the key0 is pressed, controlling the laser rangefinder to emit the laser. When the laser arrives at the measured object, the laser is bouncing back, when the laser ranging module receives the 
reflected laser, and through the mixing distance. The controller controls the liquid crystal display module to display the measured results. When the distance is measured, the controller controls the voice broadcast module to determine whether the most significant bit of the measured distance is ten or a bit, whether to add "ten" after the number, and then broadcast the subsequent numbers.

\section{Acknowledgement}

This research was financially supported by the College Science and technology project of Shandong Province (J15LN59, J16LN75 ,J16LN78)and QingDao huanghai unversity project(2016kj04).

\section{References}

[1] Li Junfeng, Ruan Linlin, Yao Dan. The Principle and Scheme of a Portable Laser Rangefinder [J] .Journal of Surveying and Mapping, 2014 (1): 103-105.

[2] Xu Hengmei, Fu Yongqing. Phase method laser ranging system [J]. Journal of Applied Science, 2010, (06): 34-35.

[3] Jiang Zhilin .STM32 single-chip principle and hardware circuit design [J]. Digital Technology and Application, 2015, (10): 56-57.

[4] Lu Youliang. STM32-based embedded system theory and design [M]. Beijing: Machinery Industry Press, 2014: 32-34.

[5] Tang Lili, Huang Wei. TM32-based FSMC interface driver TFT color screen design [J]. Modern Electronics Technology, 2013,14 (20): 139-141. 